\title{
Control efficiency improvement of an electro-hydraulic winch
}

\author{
I.C. Duțu ${ }^{1}$, C. Frățilă ${ }^{2}$, T. Axinte ${ }^{2}$, M.G. Munteanu ${ }^{1}$, L. Calancea ${ }^{2}$, M. Diaconu ${ }^{2}$, \\ C. Drăgan ${ }^{3}$ \\ ${ }^{1}$ Politehnica University of Bucharest \\ ${ }^{2}$ Research and Innovation Center for Navy \\ ${ }^{3}$ Constanța Maritime University
}

Corresponding author: T. Axinte, tibi_axinte@yahoo.com

\begin{abstract}
The paper presents a study regarding electro-hydraulic control systems for drive winches, a structural part of LARS (Launch and Recovery Systems). In the Introduction section of the paper, the authors present the domains of the research vessel. Furthermore, there is presented the importance of launch and recovery systems $(L A R S)$ and drive winches on the deck of a research vessel. The launch and recovery systems (LARS) using drive winches are installed on the stern of the research vessel. Further in the paper, the authors present the results of studying three simplified systems that use electric, hydraulic and electro-hydraulic driving solutions. Furthermore, there are presented comparative advantages of using this three types of drive winches. At the end of the paper, the authors perform an analysis of the electro-hydraulic systems for the drive winch, using a modelling and simulation software. Each schematic is presented along with its components. Moreover, the authors mention that all the schematics presented in this paper are modelled using FluidSim software from FESTO. In this case, only three mathematic relations are used in the paper: the Cauchy momentum (convective form), the incompressible Euler relations and the pressure losses in the hydraulic and electro-hydraulic drive winch.
\end{abstract}

Keywords: system, drive, winch, hydraulic, sketch.

\section{Introduction}

When attempting to model the decision-making process related to the design of a research vessel, it appears that this is very difficult for operators, [1].

In the case that the authors present in this paper, it is shown the best 3D model designed for a research vessel with $L A R S$, as shown in figure 1 .

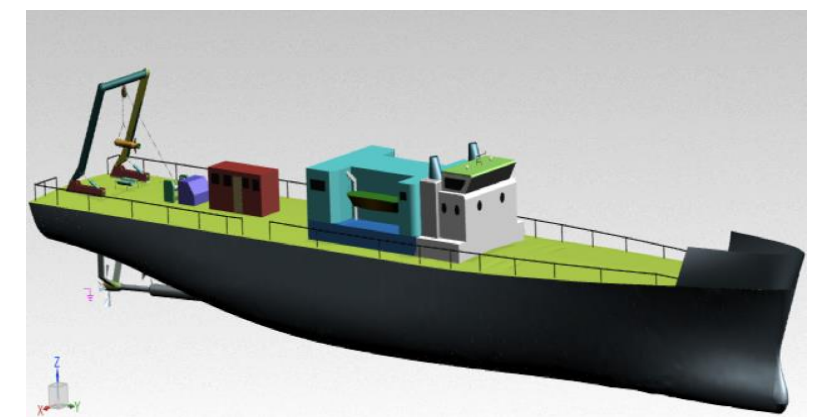

Figure 1. Research vessel with $L A R S$. 
Research vessels use complex driving installations that are mounted in the stern of the vessel. These hydraulic installations mainly consist of: a base layer, an A-frame or a L-frame, a clump weight, a hydraulic power-pack and drive winches, as shown in figure 2.

The deck equipment of a research vessel is mainly used for launch or recovery of underwater devices $(L A R S)$ such as: autonomous underwater vehicles $(A U V)$, remotely operated underwater vehicles $(R O V)$, mini submarines (midget submarines), diving baskets and gliders, [2].

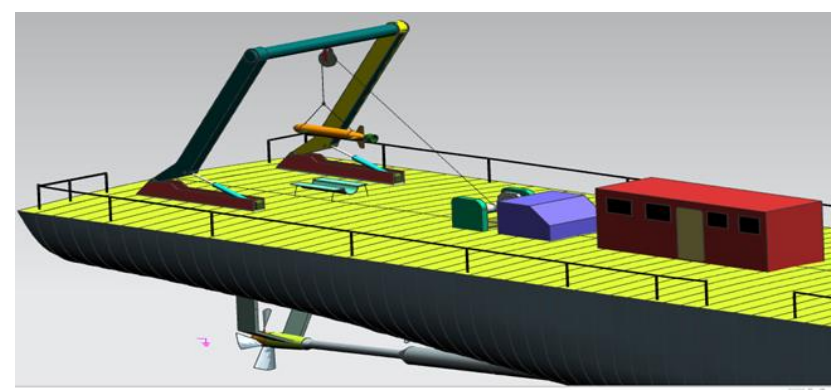

Figure 2. $L A R S$ with $A U V$.

The commissioning of drive winch mounted in the chamber of motor is given in figure 3.

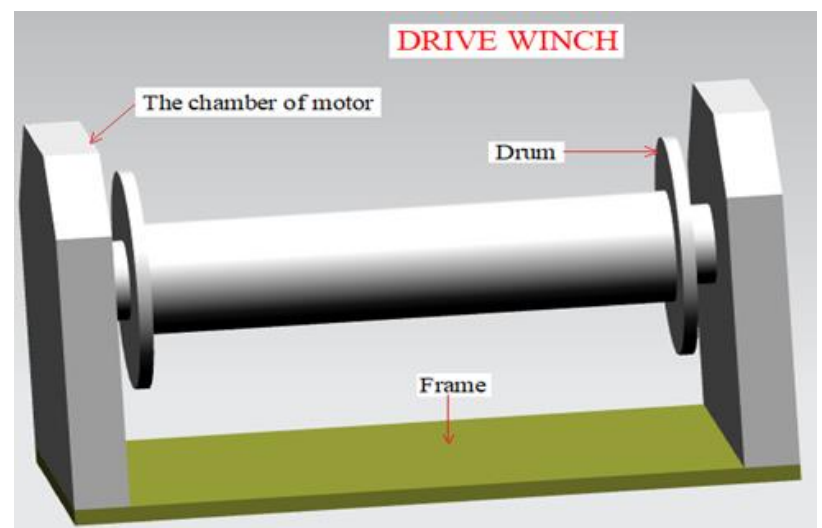

Figure 3. Schematic commissioning of the drive winch.

The connection between the drive winch and the underwater vehicle is made using wire ropes, as shown in figure 3 .

\section{Hydraulic driven winch}

In practice, when referring to a research vessel, there are used three types of driven winches, as follows:

- $D C$ electric driven winches;

- hydraulic driven winches;

- electro-hydraulic driven winches.

On the research vessel that the authors have studied, use of $D C$ electric driven winches are an alternative to using hydraulic and electro-hydraulic driven winches. $D C$ electric driven winches are gaining a lot of terrain due to their reduced energy consumption.

Mainly, a $D C$ electric drive winch that can be mounted on a research vessel is usually made of the following components:

- $\quad D C$ inductor motor;

- variable frequency drive;

- sensor, used for providing feedback;

- reduction gearbox;

- control system;

- braking system;

- cooling system. 
From a general point of view, the advantages of using $D C$ electric driven winches are:

- less noise when working in normal operating mode;

- advanced precision control;

- reduced maintenance costs;

- no hydraulic oil required;

- reduced energy consumption.

Hydraulic driven systems used for research vessels are similar to electro-hydraulic winches, excepting the HPU (hydraulic power unit), because this is only being used to power various hydraulic motors, [3].

The authors consider that there are four reasons to use a hydraulic driven winch instead of using a $D C$ electric driven winch:

- it is a very well-known fact that most equipment and machinery comply to a very simple rule: the more powerful they are, the heavier their weight. However, this is not the case of using a hydraulic driven winch that often weights less than its electrical counterparts;

- $D C$ electric driven winches often overheat if are used for a long period of time, requiring the operator to power them properly and to provide a sufficient cooling period of time before it can be used again. However, hydraulic driven winches can be continuously operated without having any concern regarding the overheating;

- hydraulic driven winches do not imply the risk in which electric motor's stall can occur while using $D C$ electric driven systems, therefore creating a serious danger due to the high tension value that can be induced in the wire cable when lifting.

The Cauchy momentum equation, given in its convective form, is valid for the fluidic hydraulic driven winches, [4]. In this case, relation of the convective form can be written as:

$$
\rho \frac{D u}{D t}=g \rho-\nabla p+\nabla \tau
$$

In equation (1), there are given:

- $\frac{D u}{D t}$, material derivative. It can be defined as: $\frac{\partial}{\partial t}+g \rho$;

- $u$, flow velocity;

- $\rho$, fluid density;

- $\nabla$, Hamilton operator;

- $t$, time;

- $\tau$, stress tensor;

- $g$, is the body acceleration action on the continuum mechanics:, gravity, inertial acceleration and so on.

In equation (1), as given above, it can be seen that when assuming an inviscid fluid (in which the viscosity of the fluid is equal to zero) its convective form reduces to the incompressible Euler equations.

Referring to the incompressible Euler equations and taking into consideration the case of density being constant in time and uniform in space, it can be written:

$$
\left\{\begin{array}{l}
\frac{D u}{D t}=g-\nabla w \\
\nabla u=0
\end{array}\right.
$$

In equation system (2) it is given $w$ as the specific thermodynamic work (having the sense of per unit mass) and the internal source term. 
The hydraulic devices used for the commissioning of the drive winch include the following components, as given in table 1 .

Table 1. Main components of the hydraulic circuit.

\begin{tabular}{lc}
\hline Description & Number of components \\
\hline Pumping unit & 1 \\
$4 / 3$ way directional valve & 1 \\
Throttle check valve $1 \& 2$ & 2 \\
Hydraulic motor 1 & 1 \\
$T 1$, manual acting lever & 1
\end{tabular}

As can be seen in figure 4, the hydraulic system will operate if $T 1$ lever is manually acted on the 4/3 way valve. Regarding the hydraulic motor 1 , its shaft can be rotated clockwise or counterclockwise according to the manual command issued.

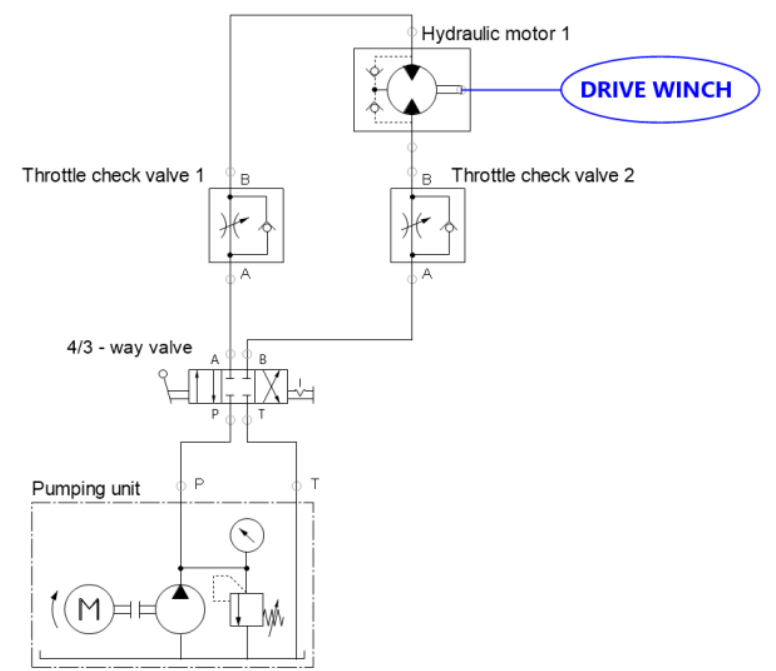

Figure 4. Schematic of the hydraulic devices with drive winch.

If the operator will pull the $T 1$ lever onto the valve's right side, then the hydraulic motor shaft will rotate clockwise, as shown in figure 5.

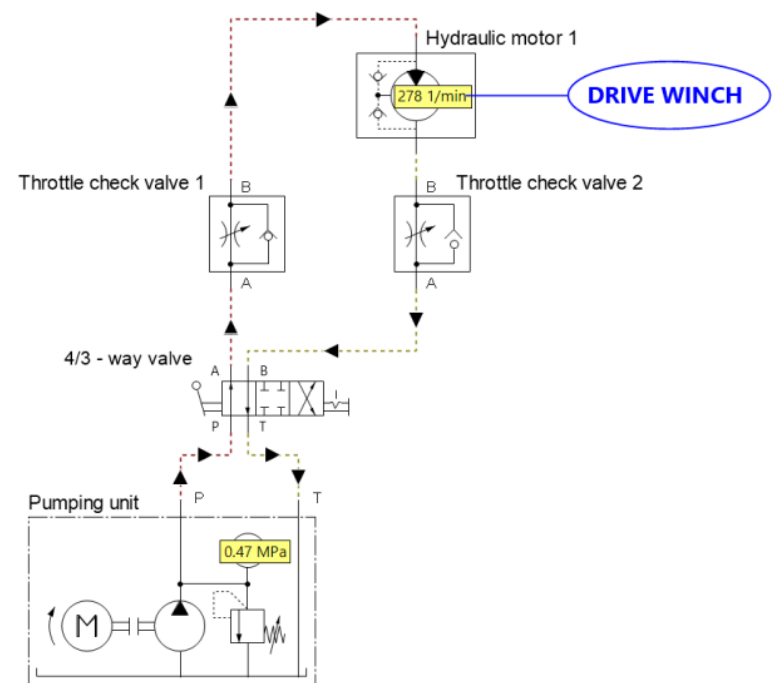

Figure 5. Hydraulic motor 1 with clockwise rotation of the shaft. 
However, if the $T l$ lever is pulled onto to the valve's left side, then the shaft of the hydraulic motor 1 will rotate counterclockwise, as shown in figure 6.

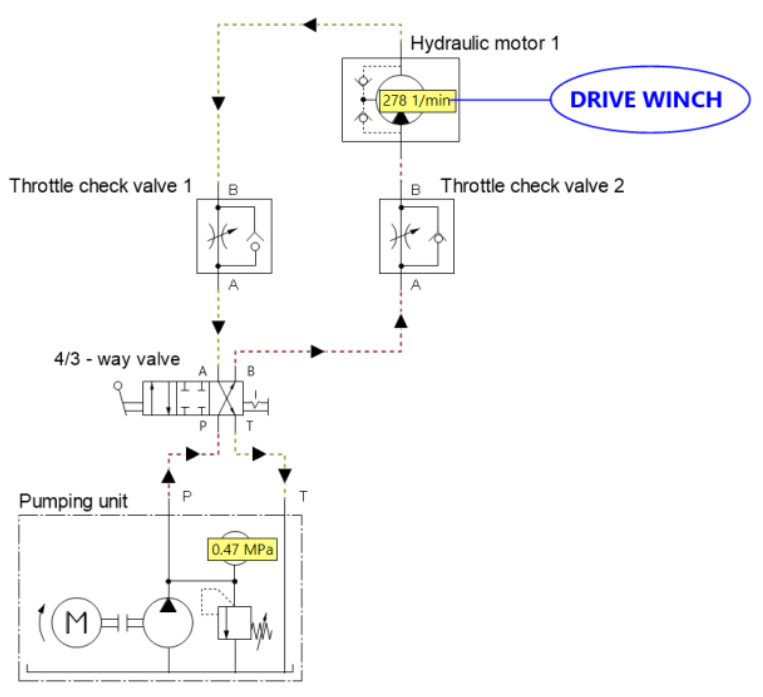

Figure 6. Hydraulic motor 1 with counterclockwise rotation of the shaft.

The rotational speed $(\mathrm{rpm})$ and the flow rate $(q)$ of the hydraulic motor 1 , are represented in two diagrams, as can be seen in figure 7 .

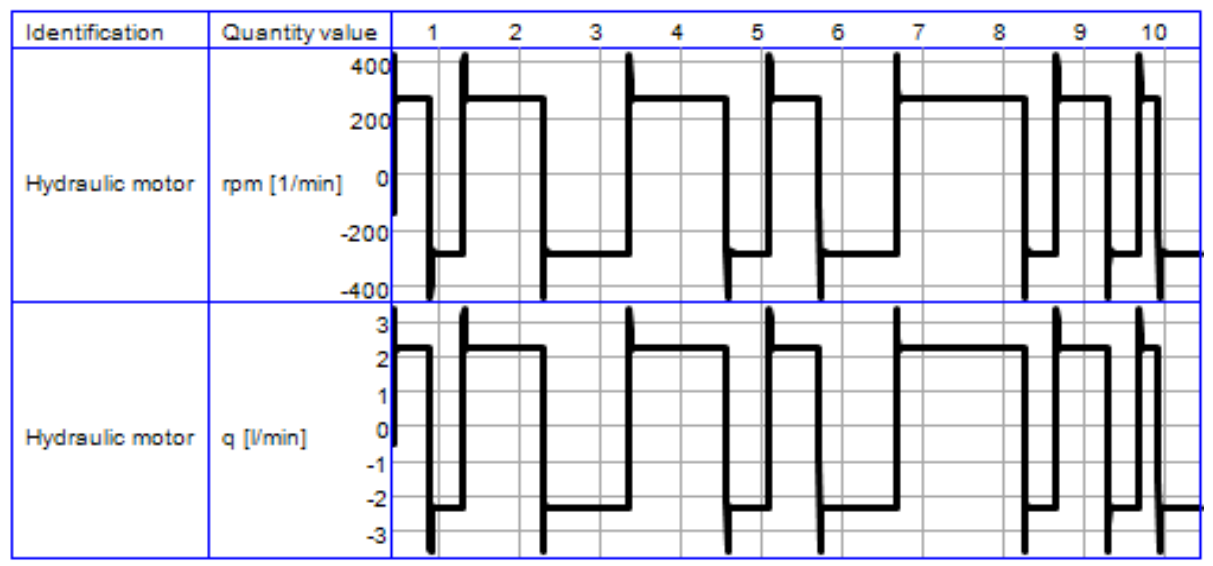

Figure 7. Functional diagrams of the hydraulic motor 1.

\section{Electro-hydraulic driven winch}

Electro-hydraulic driven winches are used in the marine environment for many decades, [5].

The electro-hydraulic driven winches are preferred to be used, due to their:

- adaptability to various operating conditions;

- relatively simple to operate and to maintain;

- ease of precision control;

- high reliability.

Mainly, the electro-hydraulic winches are made up of the following components:

- hydraulic motor;

- hydraulic power unit $(H P U)$;

- cooling system;

- braking system;

- $\quad$ sensor feedback;

- control system. 


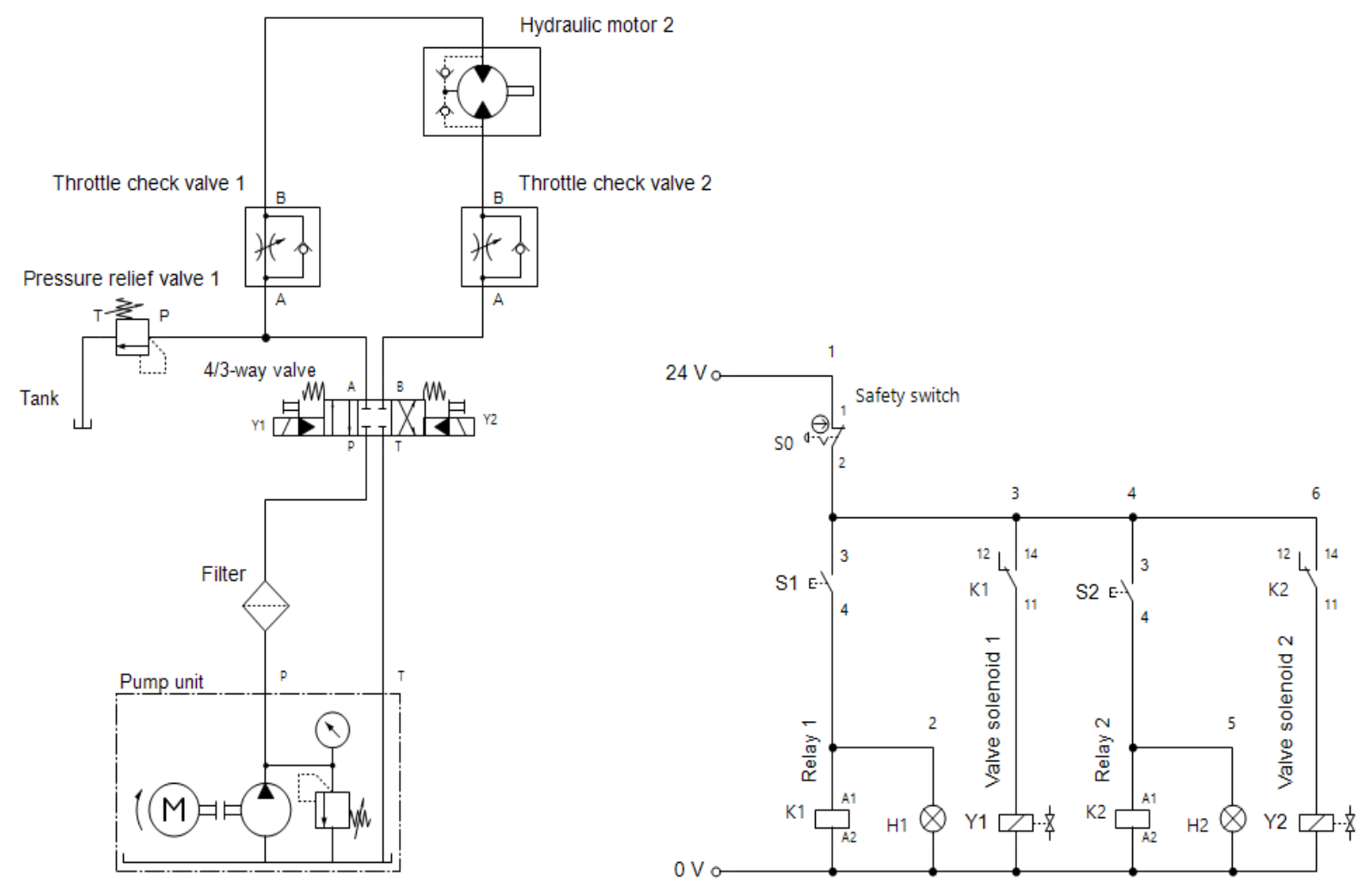

Figure 8. Schematic of the electro-hydraulic system with drive winch.

The electro-hydraulic system model presented by the authors can be seen as a simple installation, having a $24 \mathrm{~V}$ DC power supply, [6].

In fact, there are electro-hydraulic systems that use supply voltages between 220 and $480 \mathrm{~V} \mathrm{AC}$, while the hydraulic motor can achieve a maximum power rating of $1400 \mathrm{~W}$, being capable to raise or lower an underwater installation of $981000 \mathrm{~N}$ weight, having a rope capacity between 20 and $1000 \mathrm{~m}$, [7].

Table 2. Main components of the electro-hydraulic circuit.

\begin{tabular}{lc}
\hline Description & Number of components \\
\hline Hydraulic oil tank & 3 \\
Oil filter & 1 \\
$4 / 3$ way directional valve & 1 \\
Throttle check valve $1 \& 2$ & 2 \\
Hydraulic motor 2 & 1 \\
Pressure relief valve 1 & 1 \\
Valve solenoid & 2 \\
Pushbutton, $N O$ contact & 2
\end{tabular}

The hydraulic system will operate if the $S 1$ button is pressed, therefore energizing the $Y 1$ valve solenoid of the $4 / 3$ way directional valve, as shown in figure 9. If the $S 1$ button will be pressed, then the shaft of the hydraulic motor 2 will rotate clockwise, [8]. 


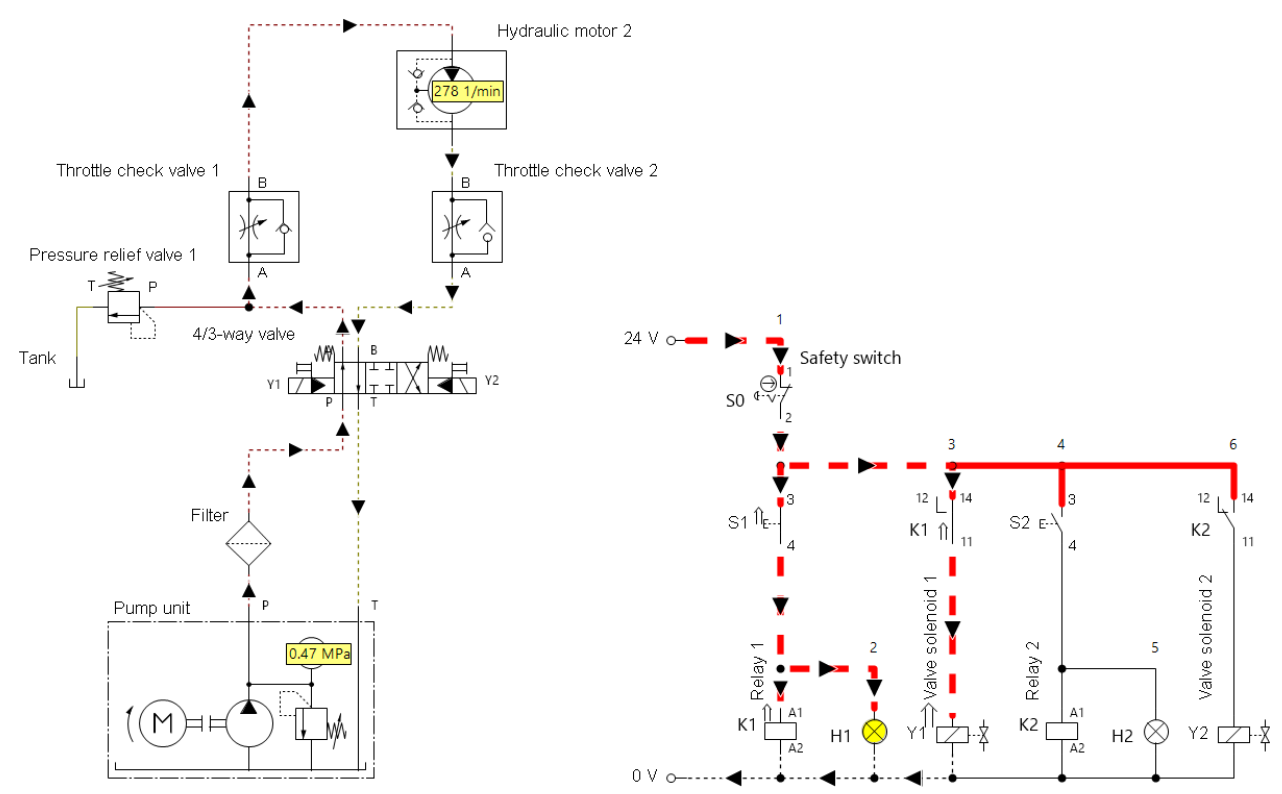

Figure 9. Hydraulic motor 2 with clockwise rotation of the shaft.

Afterwards, if the $S 2$ button will be pressed, after releasing $S 1$, the shaft of the hydraulic motor 2 will rotate counterclockwise, as seen in figure 10.

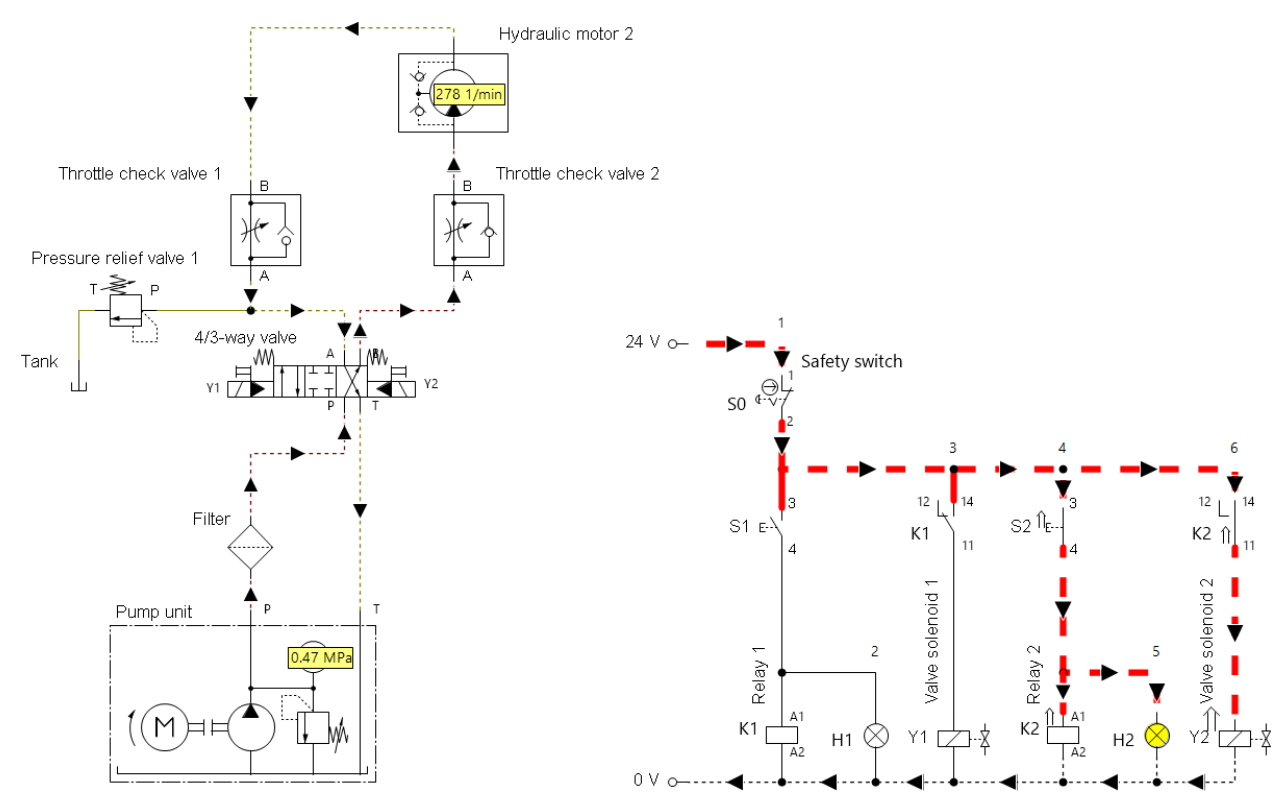

Figure 10. Hydraulic motor 2 with counterclockwise rotation of the shaft.

The revolution rate $(\mathrm{rpm})$ and the flow rate $(q)$ of the hydraulic motor 2, are represented in the following two diagrams, as given in figure 11.

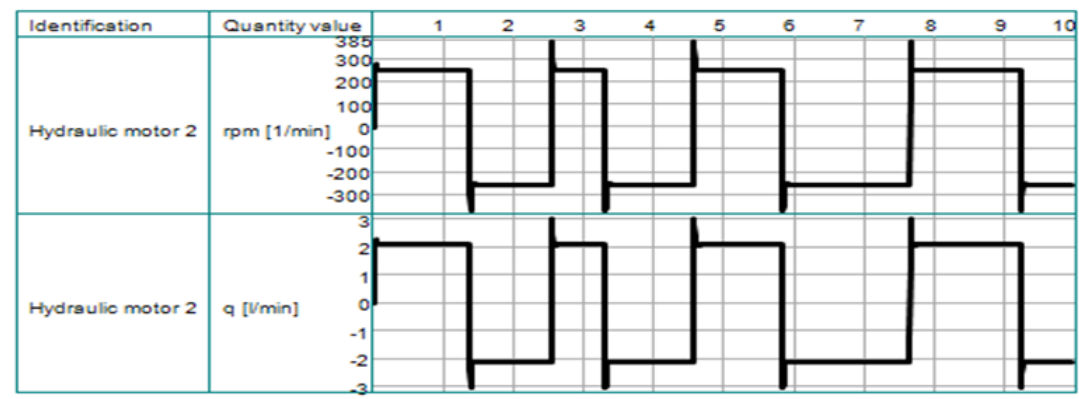

Figure 11. Functional diagrams of the hydraulic motor 2. 


\section{Conclusions}

Hydraulic, electric or electro-hydraulic driven winches, each have advantages and disadvantages while using on a research vessel. Selection of one winch drive system should be based on the intended use and should be compared with weighted criteria. Considering the case of electro-hydraulic drive winches, the authors consider that it is easier to diagnose them and repair its components, than the other types of winches.

Pressure losses generated by the hydraulic motor will affect the overall use of the drive winch. Thus, the drive winch will be blocked if there is detected a high pressure loss at the hydraulic motor level. This can be possible during the process of recovery and launch of an underwater vehicle. In these cases, the safety of the research vessel may be endangered. Nevertheless, there are known seven reasons to perform preventive maintenance on the drive which systems, as follows:

- protection of people and working equipment;

- reliable performance;

- lower total cost of ownership;

- improved spare parts management;

- higher energy efficiency;

- compliance with actual legal requirements.

The electro-hydraulic driven winches denote certain advantages according to the following criteria: installation cost, maintainability, reliability, tension, speed, load control, thermal management. Moreover, this type of winches can withstand severe functional weather conditions such as: storms, strong winds, high waves, etc. Therefore, many newly constructed research vessels are provided with electro-hydraulic driven winches. The authors will furthermore investigate the improvement of the control efficiency in an electro-hydraulic driven winch, approaching the use of another method for flow regulation than the one presented in this paper. Another future step is to integrate a PLC (Programmable Logic Controller) into the control circuitry, providing much more flexibility.

\section{Acknowledgement}

This research was partially supported by the Executive Unit for Financing Higher Education, Research, Development and Innovation, UEFISCDI, under the PNCDI III - Programme 2, sub-programme 2.1, submission code PN-III-P2-2.1-PTE-2019-0446, funding contract no. 53PTE/23.09.2020, project title "Hydrophilic auto-chassis for high energy efficiency operation of interchangeable equipment intended for performing public utility work", acronym ASHEUP, research direction 3.Econano-technologies and advanced materials, subdomain Advanced materials.

\section{References}

[1] B.C. BELEV: Information Characteristics of a Man-Operator for Ensuring the Vessel's Safety of Navigation, Journal ENC-GNSS, (2010).

[2] S.V. PATURCA, R.G. DAMIAN, N. JULA: Autonomous Underwater Vehicles - achievements and current trends, Scientific Bulletin of Navala Academy, Vol. 21, pp. 85-89, Constanta, Romania, 2018.

[3] L. SCHIMDT, H. C. PENDERSEN, V. H. DONKOV, T.O. ANDERSEN: Analysis \& Control of a Self-Contained Hydraulic Winch Drive, Symposium on Fluid Power \& Motion Control, Proceeding of Bath/ASME, UK, September 12-14, 2018.

[4] V. CRISTEA, M. DEGERATU, N.I. ALBOIU: Rotational Flow for Real Incompressible Fluids, Scientific Bulletin of the Technical University of Civil Engineering, Series: Mathematical Modelling in Civil Engineering, issue 3, pp. 24-36, 2006.

[5] ***, https://www.festo-didactic.com/ro-ro/, last accessed on 15 September 2021.

[6] Y.F. HOU, D. M. WANG, Q.R. MENG, B. DU: Dynamic Analysis of a New Type of HydroViscous Drive Winch, Advanced materials Research, ISSN: 1662-8985, vols. 328-330, pp. 2243-2247, doi: 10.4028/www.scientific.net/AMR.328-330.2243, Trans Tech Tech Publication Ltd, 2011.

[7] I. GUȚĂ, C. DUMITRESCU, I. LEPĂDATU, C. CRISTESCU: Experimental identification of electrohydraulic servomechanisms with virtual instruments technique, Hidraulica Revue, no.3/2010, pp. 49-56, ISSN 1453-7303, 2010. 